\title{
Monitoring cardiovascular function in infants with chronic lung disease of prematurity
}

\section{S H Abman}

In addition to persistent airways disease, survivors of premature birth with chronic lung disease are at risk of cardiovascular sequelae, including pulmonary hypertension, systemic hypertension, left ventricular hypertrophy, and exercise intolerance. The major treatment of pulmonary hypertension is supplemental oxygen, but drugs such as calcium channel blockers may also be required. The use of inhaled nitric oxide for its long term management is being investigated

$\ldots \ldots \ldots \ldots \ldots \ldots \ldots \ldots$

remature birth with injury to the immature lung disrupts the normal sequence of lung growth and leads to the development of severe chronic lung disease (CLD) or bronchopulmonary dysplasia (BPD). CLD is the chronic pulmonary disease of infancy that follows ventilator and oxygen therapy for neonatal respiratory failure shortly after birth. ${ }^{1}$ It is typically defined by the presence of persistent respiratory signs and symptoms, the need for supplemental oxygen to treat hypoxaemia, and abnormal chest radiograph at 36 weeks corrected age. However, since the introduction of surfactant therapy and new ventilator strategies, the clinical course and outcomes of premature babies with respiratory distress syndrome (RDS) have changed considerably. ${ }^{23}$ There is growing recognition that infants with persistent lung disease after premature birth have a different clinical course and pathology from that traditionally observed in infants dying from CLD in the era before the use of surfactant. Now, infants with CLD are mainly premature newborns who are far less mature and with much lower birth weight than previously described. In addition to its changing epidemiology, the nature of CLD has evolved as well, such that pathological signs of severe lung injury with striking fibroproliferation are less common. More typically, infants with CLD now have less severe acute respiratory disease early, and, at autopsy, lung histology shows more uniform and milder regions of injury, with signs of impaired alveolar and vascular growth becoming more prominent. ${ }^{2}$ This "new BPD" is only beginning to be characterised, especially with regard to implications for long term outcome. Thus, most of our current follow up data are actually based on patients from the era before surfactant, with only limited studies describing the clinical course of patients who have reached adolescence and young adulthood.

Although most studies of CLD have focused primarily on airways disease, infants with CLD are also at high risk of cardiovascular sequelae, such as pulmonary hypertension, impaired gas exchange due to abnormal vasoregulation, exercise intolerance, systemic hypertension, left ventricular hypertrophy (LVH), systemic to pulmonary collateral vessels, and others. ${ }^{4}$ Unfortunately, there are few published studies that relate the incidence and severity of these cardiovascular problems to the "new BPD." Despite this limitation, this article will briefly review our current approach towards the monitoring of cardiovascular disease in infants with CLD, and discuss related problems of long term follow up. Oxygen therapy and its monitoring are discussed in the preceding article by Kotecha and Allen.

\section{THE PULMONARY CIRCULATION IN CLD}

In addition to adverse effects on the airway and distal airspace, acute lung injury also impairs growth, structure, and function of the developing lung circulation after premature birth (fig 1). Experimental studies have clearly shown that endothelial cells are particularly susceptible to oxidant injury caused by hyperoxia or inflammation. The small pulmonary arteries also undergo striking changes, including smooth muscle cell proliferation, precocious maturation of immature pericytes into mature smooth muscle cells, and incorporation of fibroblasts into the vessel wall. ${ }^{6-8}$ Structural changes in the lung vasculature contribute to high pulmonary vascular resistance (PVR) through narrowing of the vessel diameter and decreased vascular compliance. In addition to these structural changes, the pulmonary circulation is further characterised by abnormal vasoreactivity, which also increases PVR.' Finally, decreased angiogenesis may further limit vascular surface area, causing further elevation of PVR, especially in response to high cardiac output with exercise or stress (fig 1).

Overall, early injury to the lung circulation leads to the rapid development of pulmonary hypertension, which contributes greatly to the morbidity and mortality of severe CLD. Even in the earliest reports of CLD, pulmonary hypertension and cor pulmonale were recognised as being associated with high mortality. ${ }^{10}{ }^{11}$ Walther et $a l^{12}$ showed that elevated pulmonary artery pressure in premature newborns with acute RDS,

Abbreviations: CLD, chronic lung disease; BPD, bronchopulmonary dysplasia; RDS, respiratory distress syndrome; PVR, pulmonary vascular resistance; iNO, inhaled nitric oxide; LVH, left ventricular hypertrophy; ECG, electrocardiogram 


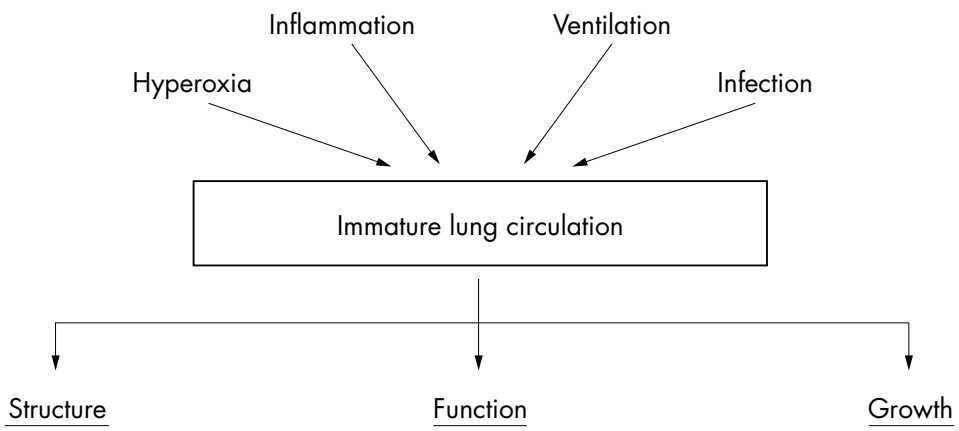

- Smooth muscle hyperplasia

- Fibroblast incorporation

- Adventitial thickening due to increased collagen, matrix
- Impaired vasodilation

- Increased hypoxic constriction

- Enhanced myogenic response (?)

- Abnormal metabolic function
- Decreased artery number

- Decreased surface area

- Reduced alveolisation (?)

Figure 1 Pulmonary circulation in chronic lung disease.

as determined from serial echocardiograms, was associated with severe disease and high mortality. Previous studies have also shown that persistent echocardiographic evidence of pulmonary hypertension beyond the first few months of life is associated with up to $40 \%$ mortality in infants with CLD. ${ }^{13}{ }^{14}$ High death rates have been reported in infants with CLD and pulmonary hypertension who require prolonged support with mechanical ventilation. ${ }^{15}$ Although it is possible that pulmonary hypertension is a marker of more advanced lung disease, high PVR clearly contributes to the pathophysiology of infants with severe CLD, causing poor right ventricular function, impaired cardiac output, limited oxygen delivery, worsened pulmonary oedema, and perhaps a higher risk of sudden death. ${ }^{4}$

"Early injury to the lung circulation leads to the rapid development of pulmonary hypertension, which contributes greatly to the morbidity and mortality of severe CLD."

Physiological abnormalities of the pulmonary circulation in CLD include elevated PVR and abnormal vasoreactivity, as evidenced by the considerable vasoconstrictor response to acute hypoxia. $^{59}$ As studied during cardiac catheterisation, acute exposure to hypoxia causes large elevations in pulmonary artery pressure, even in infants with modest basal levels of pulmonary hypertension. ' This study further showed that the degree of hypoxia that increases pulmonary hypertension can be fairly modest in many patients, and that achieving oxygen saturations above $92-94 \%$ was effective in lowering pulmonary artery pressure. A recent study (the STOP-ROP trial) ${ }^{16}$ examined the role of oxygen therapy in retinopathy of prematurity, and suggested that infants who were treated with hyperoxia to maintain high oxygen saturations had a higher risk of CLD than infants treated with supplemental oxygen that targeted lower saturations. However, interpretation of these data for use in the prevention or treatment of pulmonary hypertension is difficult, as the targeted ranges for the low and high oxygen saturations fell between typical recommendations for oxygen therapy. That is, the current recommendation for treatment of patients with CLD and pulmonary hypertension is to avoid oxygen saturations below $92 \%$, and to maintain levels of $94-96 \%$. Whether the risk of attaining higher oxygen saturations is to induce more lung injury remains unconfirmed, but the adverse effects of hypoxaemia are clear. Strategies that maintain lower pulmonary artery pressure or limit injury to the pulmonary vasculature during the treatment of acute RDS may attenuate the subsequent development or contribution of pulmonary hypertension to CLD.
In addition to pulmonary hypertension, clinical studies have also shown that metabolic function of the lung circulation is impaired, as reflected by the lack of pulmonary clearance of circulating noradrenaline (norepinephrine) across the lung. ${ }^{17}$ For example, the lung circulation normally clears $20-40 \%$ of circulating noradrenaline during a single passage through the lung, but infants with severe CLD had a net production of noradrenaline across the pulmonary circulation. Whether impaired metabolic function of the lung contributes to the pathophysiology of CLD by increasing circulating catecholamine levels, or if it is simply a marker of severe pulmonary vascular disease, remains unknown. It has been speculated that high catecholamine levels may lead to LVH or systemic hypertension, which are known complications of CLD (see below).

\section{"Strategies that maintain lower pulmonary artery pressure or limit injury to the pulmonary vasculature during the treatment of acute RDS may attenuate the subsequent development or contribution of pulmonary hypertension to CLD."}

As described above, the "new BPD" is characterised by a striking reduction in alveolar growth, and it is also likely that vascular growth is decreased. Impaired angiogenesis reduces vascular surface area, and may increase the risk of pulmonary hypertension, cause further impairment of gas exchange (especially with respiratory infections, pulmonary oedema, or stress), and contribute to reduction of exercise capacity in older children. ${ }^{18}$ Mechanisms that coordinate vascular and alveolar growth in CLD are poorly understood, but hyperoxia inhibits alveolar number and reduces the ratio of arteries to alveoli in neonatal rats. ${ }^{78}$ Disruption of key signals between airway epithelium and endothelial cells during development is likely to alter vascular growth, decreasing arterial number. Recent animal studies support this hypothesis. Treatment of newborn rats with antiangiogenesis drugs (thalidomide and fumagillin) and a vascular endothelial growth factor receptor inhibitor inhibits vascular growth and decreases alveolisation. ${ }^{19}$ These findings suggest that angiogenesis is necessary for alveolisation during normal lung development, and that injury to the developing pulmonary circulation during a critical period of lung growth can contribute to lung hypoplasia. Therefore protecting the pulmonary vasculature from injury may not only lower PVR and improve gas exchange, but it may also enhance distal lung growth and improve long term outcome. Extensive laboratory and some clinical studies suggest that inhaled nitric oxide (iNO) lowers PVR and improves oxygenation in patients with pulmonary hypertension in different settings, including premature 
infants with severe RDS and CLD. ${ }^{20}$ However, there are persistent concerns about potential toxicity and adverse effects of iNO therapy in the premature infant. Experimental data have suggested that iNO therapy may be "lung protective" in several animal models, including premature lambs with RDS. $^{21}$ Whether iNO therapy has a potential role in the prevention of pulmonary vascular injury in premature newborns at risk of CLD is not known. A multicentre clinical trial of low dose iNO therapy ( $5 \mathrm{ppm}$ ) was performed in severely ill premature newborns with RDS who had considerable hypoxaemia despite surfactant therapy-that is, a/A $\mathrm{O}_{2}$ ratio $<0.10$-with an estimated mortality of $53 \%{ }^{22}$ In this study, iNO acutely improved $\mathrm{PaO}_{2}$, but did not improve survival. Notably, there was no increase in the incidence or severity of intracranial haemorrhage or CLD, and the duration of mechanical ventilation was reduced. Based on findings, a multicentre trial is now underway to determine whether early treatment with low dose iNO therapy will prevent the early inflammatory changes that contribute to CLD, and protect the pulmonary circulation from injury during this critical time period.

"These findings suggest that angiogenesis is necessary for alveolisation during normal lung development, and that injury to the developing pulmonary circulation during a critical period of lung growth can contribute to lung hypoplasia."

\section{ASSOCIATED CARDIOVASCULAR ABNORMALITIES IN CLD}

Other cardiovascular abnormalities associated with CLD include LVH, systemic hypertension, and the development of prominent systemic to pulmonary collateral vessels. ${ }^{43}$ An early report described infants with severe CLD and LVH in the absence of right ventricular hypertrophy, and suggested that left ventricular dysfunction may contribute to recurrent oedema in CLD. ${ }^{24}$ Steroid treatment can cause LVH, and in this setting, LVH tends to be transient, resolves with cessation of steroid treatment, and is of uncertain clinical importance.

A high incidence of systemic hypertension has also been recognised as a cardiovascular complication of CLD, but its cause remains obscure. ${ }^{25}$ Systemic hypertension may be mild, transient, and respond readily to pharmacological treatment; however, the rise in blood pressure can be striking in some cases. On occasion, further evaluation of such infants shows considerable renal vascular or urinary tract disease. Whether the high incidence of systemic hypertension in CLD reflects altered neurohumoral regulation or increased catecholamines, angiotensin, or antidiuretic hormone levels is not known.

Prominent bronchial or other systemic to pulmonary collateral vessels were noted in early morphometric studies of infants with CLD, and can be readily identified in many infants during cardiac catheterisation. ${ }^{4}{ }^{15}$ Although these collateral vessels are generally small, large collaterals may contribute to appreciable shunting of blood flow to the lung, causing oedema and the need for higher $\mathrm{FIO}_{2}$. Collateral vessels have been associated with high mortality in some patients with severe CLD who also had severe pulmonary hypertension. ${ }^{15}$ Some infants have improved after embolisation of large collateral vessels, as reflected by a reduced need for supplemental oxygen, ventilator support, or diuretics, but the actual contribution of collateral vessels to the pathophysiology of CLD is poorly understood.

\section{CLINICAL APPROACH TO CARDIOVASCULAR MONITORING}

As briefly reviewed, cardiovascular sequelae, especially pulmonary hypertension, can contribute significantly to the clinical course of infants with severe CLD. A general approach to
General suggestions for cardiovascular monitoring of infants with severe CLD

- Maintain oxygen saturations $>92 \%$ if clinically stable without pulmonary hypertension; $94-96 \%$ for infants with pulmonary hypertension

- Monitoring of oxygen requirements necessitates prolonged pulse oximetry-for example, awake, asleep, and during feeding

- Routinely monitor blood pressure (at least weekly for inpatients, or at each outpatient visit)

- Serial echocardiograms every two to three months in patients with CLD who require prolonged ventilator and oxygen therapy, or with pulmonary hypertension

- Cardiac catheterisation for patients with severe CLD, persistent pulmonary hypertension, in order to: rule out anatomical cardiac disease or pulmonary vein stenosis or occlusion; assess severity of pulmonary hypertension; test reactivity to oxygen or vasodilators; determine whether large systemic to pulmonary collaterals are present

cardiovascular monitoring is outlined. Most patients with "resolving" CLD show progressive increases in growth parameters and follow their growth curves for body weight and length. ${ }^{26}$ Patients who show evidence of poor growth, persistent right ventricular hypertrophy by electrocardiogram (ECG), or additional signs of pulmonary hypertension by ECG require more extensive studies. Firstly, prolonged or intermittent periods of acute hypoxia are the most likely causes of persistent pulmonary hypertension in CLD. Clinical evaluations of such patients should include prolonged measurements of oxygen saturation by pulse oximeter while awake, asleep, and during feeds. ${ }^{4}$ Brief assessments of oxygenation ("spot checks") are not sufficient for decisions on the level of supplemental oxygen needed. As described above, targeting oxygen saturations to $92-94 \%$ should be sufficient to prevent the adverse effects of hypoxia in most infants, without increasing the risk of additional lung inflammation and injury. A sleep study may be necessary to determine the presence of noteworthy episodes of hypoxia and whether hypoxaemia has predominantly obstructive, central, or mixed causes. It is not uncommon to uncover unsuspected causes of airway obstruction in patients with CLD and persistent pulmonary hypertension, such as tonsillar and adenoidal hypertrophy, vocal cord paralysis, subglottic stenosis, tracheomalacia, and others. Additional studies that may be required include flexible bronchoscopy for the diagnosis of anatomical and dynamic airway lesions (such as tracheomalacia) that may contribute to hypoxaemia and poor clinical responses to oxygen therapy. Growth failure during home oxygen therapy may be the result of poor parental compliance or premature discontinuation of supplemental oxygen, as previously reported.$^{27}$ In this study, nearly one third of families in a home oxygen programme had prematurely discontinued oxygen therapy.

Serial assessments of pulmonary hypertension should generally include serial ECGs in infants with mild CLD who are rapidly improving. Although ECG studies are easiest to obtain, some patients can have significant right ventricular dysfunction and pulmonary hypertension despite minimal findings or normal ECG studies. As a result, we recommend serial echocardiograms in patients with moderate or severe CLD, or with a past ECG showing the presence of right ventricular hypertrophy. Previous work suggests that, with adequate oxygen therapy and related interventions, right ventricular hypertrophy should resolve in most children before 6-12 months of age. Cardiac catheterisation is reserved for patients with the most severe disease which has not responded to oxygen therapy. The purpose of cardiac catheterisation is to rule out anatomical cardiac lesions, especially associated with left to right shunting of blood. Past experience suggests that 
infants with CLD do not tolerate even relatively small atrial or ventricular level shunting, and are at high risk of developing more severe pulmonary hypertension than other patients. This may be because of the decrease in vascular surface area relative to body size and cardiac output in CLD, and the fact that even mild increases in pulmonary blood flow may represent relatively high shunt. As a result, we advise earlier closure of shunt lesions in patients with CLD, especially with the growing development of non-invasive approaches. In addition, cardiac catheterisation is useful for the following: to ensure the absence of pulmonary vein stenosis or venoocclusion; to define the level and severity of pulmonary hypertension; to assess vasoreactivity to increased oxygen tension or to assess the response to vasodilator treatment; and to determine whether large systemic to pulmonary collateral vessels are present. Studies of vasoreactivity are helpful to determine the relative safety-that is, lack of systemic hypotension and impaired cardiac contractility-and potential efficacy of drugs such as calcium channel blockers, which would be initiated in patients with exceptional pulmonary hypertension despite adequate oxygen therapy. I believe that treatment with calcium channel blockers should not be started without first determining the clinical response during cardiac catheterisation. Clearly, cardiac catheterisation is invasive and reserved for the most severely ill infants who develop pulmonary hypertension despite optimal medical management.

\section{CONCLUSIONS}

In addition to persistent airways disease, survivors of premature birth with CLD are at risk of cardiovascular sequelae, including pulmonary hypertension, systemic hypertension, LVH, exercise intolerance, and related clinical problems. Early monitoring with serial echocardiograms aids the selection of infants with CLD who are at high risk of cardiovascular morbidity and allows earlier initiation of treatment after a rigorous diagnostic evaluation. The major treatment of pulmonary hypertension in CLD is supplemental oxygen, but infants with persistent pulmonary hypertension despite adequate treatment may also require pharmacological treatment-for example, with calcium channel blockers. Whether iNO will prove to be useful for the long term management of pulmonary hypertension in CLD is uncertain, and several multicentre trials are underway. Several important problems persist with regard to our understanding of the pulmonary circulation in CLD. Firstly, there is a need to learn more about the changing clinical pattern of pulmonary hypertension and related cardiovascular sequelae of the "new BPD," which is primarily characterised by reduced septation and vascular surface area. We also need to learn more about the physiological mechanisms by which pulmonary vascular dysfunction impairs gas exchange and alters vascular tone and reactivity. In addition, more information is needed on the contribution of abnormal vascular growth to impaired distal airspace development, and whether treatments that protect vascular growth will improve long term outcome. As we learn more about the nature of lung injury and disruption of lung development, it has become evident that damage to the developing vessels, as well as the airway and distal airspaces, contribute to the late sequelae of CLD. Novel approaches to the protection of the immature endothelium and enhancement of lung vascular growth may decrease the risk of clinical problems, such as pulmonary hypertension.

\section{REFERENCES}

1 Northway WH, Rosan RC, Porter DY. Pulmonary disease following respiratory therapy of hyaline membrane disease: bronchopulmonary dysplasia. N Engl J Med 1967;276:357-68.

2 Jobe AH, Bancalari E. Bronchopulmonary dysplasia. Am J Respir Crit Care Med 2001;163:1723-9.

3 Hussain AN, Siddiqui NH, Stocker JT. Pathology of arrested acinar development in postsurfactant BPD. Hum Pathol 1998;29:710-17.

4 Abman SH, Sondheimer HS. Pulmonary circulation and cardiovascular sequelae of BPD. In: Weir EK, Archer SL, Reeves JT, eds. Diagnosis and treatment of pulmonary hypertension. New York: Futura, 1992:155-80.

5 Abman SH. Pulmonary hypertension in chronic lung disease of infancy. Pathogenesis, pathophysiology and treatment. In: Bland RD, Coalson JJ, eds. Chronic lung disease of infancy. New York: Marcel Dekker, 2000:619-68

6 Jones R, Zapol WM, Reid LM. Oxygen toxicity and restructuring of pulmonary arteries: a morphometric study. Am J Pathol 1985; 121:212-23.

7 Roberts RJ, Weesner KM, Bucher JR. Oxygen-induced alterations in lung vascular development in the newborn rat. Pediatr Res 1983;17:368-75.

8 Wilson WI, Mullen M, Olley PM, et al. Hyperoxia-induced pulmonary vascular and lung abnormalities in young rats and potential for recovery. Pediatr Res 1985;19:1059-67.

9 Abman SH, Wolfe RR, Accurso FJ, et al. Pulmonary vascular response to oxygen in infants with severe BPD. Pediatrics 1985;75:80-4

10 Tomashefski JF, Opperman HC, Vawter GF. BPD: a morphometric study with emphasis on the pulmonary vasculature. Pediatr Pathol 1984;2:469-87.

11 Anderson WR, Engel RR. Cardiopulmonary sequelae of reparative stages of BPD. Arch Pathol Lab Med 1983;107:6603-8.

12 Walther FJ, Bender FJ, Leighton JO. Persistent pulmonary hypertension in premature neonates with severe RDS. Pediatrics 1992;90:899-904.

13 Fouron JC, LeGuennec JC, Villemont D, et al. Value of echocardiography in assessing the outcome of BPD. Pediatrics 1980;65:529-35

14 Halliday HL, Dumpit FM, Brady JP. Effects of inspired oxygen on echocardiographic assessment of pulmonary vascular resistance and myocardial contractility in BPD. Pediatrics 1980;65:536-40.

15 Goodman G, Perkin R, Anas N. Pulmonary hypertension in infants with BPD. J Pediatr 1988;112:67-72.

16 Supplemental Therapeutic Oxygen for Prethreshold Retinopathy of Prematurity (STOP-ROP), a randomized controlled trial. Pediatrics 2000; 105:295-310.

17 Abman SH, Schaffer MS, Wiggins JW, et al. Pulmonary vascular extraction of circulating norepinephrine in infants with BPD. Pediatr Pulmonol 1987:3:386-91.

18 Mitchell SH, Teague G. Reduced gas transfer at rest and during exercise in school age survivors of BPD. Am J Respir Crit Care Med 1998;157:1406-12.

19 Jakkula M, Le Cras TD, Gebb S, et al. Inhibition of angiogenesis decreases alveolisation in the developing rat lung. Am J Physiol 2000;279:L600-7.

20 Banks BA, Seri J, Ischiripolous $\mathrm{H}$, et al. Changes in oxygenation with inhaled NO in severe BPD. Pediatrics 1999;103:610-18

21 Kinsella JP, Parker TA, Galan H, et al. Effects of inhaled NO on pulmonary edema and lung neutrophil accumulation in severe experimental HMD. Pediatr Res 1997;41:457-63.

22 Kinsella JP, Walsh WF, Bose C, et al. Randomized controlled trial of inhaled nitric oxide in premature neonates with severe hypoxemic respiratory failure. Lancet 1999;354:1061-5.

23 Apkon M, Nehgme RA, Lister G. Cardiovascular abnormalities in BPD. In: Bland RD, Coalson JJ, eds. Chronic lung disease of infancy. New York: Marcel Dekker, 2000:321-56.

24 Melnick G, Pickoff AS, Ferrer PC, et al. Normal pulmonary vascular resistance and left ventricular hypertrophy in young infants with BPD: an echocardiographic and pathologic study Pediatrics 1980;66:586-96.

25 Abman SH, Groothius JR. Pathophysiology and treatment of BPD. Current Issues. Pediatr Clin N Am 1994;41:277-315.

26 Abman SH, Accurso FJ, Koops BL. Experience with home oxygen in the management of infants with BPD. Clin Pediatr 1984;23:471-6.

27 Groothius JR, Rosenberg AA. Home oxygen promotes weight gain in infants with BPD. American Journal of Diseases in Children $1987 ; 141: 992-5$. 